INRNE-TH-94/3

\title{
Wigner quantum oscillators. Osp(3/2) oscillators
}

\author{
T. D. Palev* \\ Clausthal-Zellerfeld, Germany \\ N. I. Stoilova \\ Institute for Nuclear Research and Nuclear Energy, 1784 Sofia, Bulgaria
}

Arnold Sommerfeld Institute for Mathematical Physics, Technical University of Clausthal, 38678

\begin{abstract}
The properties of the three-dimensional noncanonical $\operatorname{sep}(3 / 2)$ oscillators, introduced in [J. Phys. A: Math. Gen. 27 (1994) 977], are further studied. The angular momentum $M$ of the oscillators can take at most three values $M=p-1, p, p+1$, which are either all integers or all half-integers. The coordinates anticommute with each other. Depending on the state space the energy spectrum can coincide or can be essentially different from those of the canonical oscillator. The ground state is in general degenerated.
\end{abstract}

\section{Introduction}

In the present paper we continue the study of the three-dimensional nonrelativistic quantum osp $(3 / 2)$ oscillators, introduced in [1]. The main algebraic feature of each such oscillator is that its position and momentum operators generate a representation of the orthosymplectic Lie superalgebra $o s p(3 / 2)$. The state space of each oscillator is an infinite-dimensional irreducible module of the Lie superalgebra (LS) $\operatorname{osp}(3 / 2)$. This result was only announced in [1]. Here we prove it. Moreover in [1] we have considered one particular oscillator, namely the one with an angular momentum $1 / 2$, stating only the energy and the angular momentum spectrum of the other possible oscillators. Here we study the physical properties of all $\operatorname{csp}(3 / 2)$ oscillators in detail, introducing an orthonormed basis, consisting of common eigenvectors of the Hamiltonian $H$, the square of the angular momentum $\mathbf{M}^{2}$ and its third projection $M_{3}$. Within each state space we compute the matrix elements of essentially all physical observables.

The motivation to introduce and study such more general, noncanonical oscillators was out-

* Permanent address: Institute for Nuclear Research and Nuclear Energy, Boul. Tsarigradsko Chausse 72, 1784 Sofia, Bulgaria; E-mail palev@bgearn.bitnet 
lined in [1]. We recall the main points. The idea belongs to Wigner [2], who observed that the Hamiltonian equations can be identical with the Heisenberg equations for position and momentum operators, which do not necessarily satisfy the canonical commutation relations (CCRs). Wigner has demonstrated this on an example of an one-dimensional harmonic oscillator, studied subsequently by several authors [3].

The question about the compatibility of the Hamiltonian equations

$$
\dot{\mathbf{p}}=-m \omega^{2} \mathbf{r}, \quad \dot{\mathbf{r}}=\frac{\mathbf{p}}{m}
$$

with the Heisenberg equations (here and throughout $[x, y]=x y-y x,\{x, y\}=x y+y x$ )

$$
\dot{\mathbf{p}}=-\frac{i}{\hbar}[\mathbf{p}, H], \quad \dot{\mathbf{r}}=-\frac{i}{\hbar}[\mathbf{r}, H]
$$

of a three-dimensional harmonic oscillator, namely of a system with a Hamiltonian

$$
H=\frac{\mathbf{p}^{2}}{2 m}+\frac{m \omega^{2}}{2} \mathbf{r}^{2},
$$

was investigated in [4] and [1]. The present paper is also in the same frame. The problem is to determine and study at least some noncanonical Wigner quantum oscillators. To be more precise with the terminology we give the following definition.

Definition 1. A triple $(\mathbf{r}, \mathbf{p}, W)$ is said to be a Wigner quantum oscillator, if it fulfills the following three conditions (we refer to them as to quantum conditions):

(i) The state space of the oscillator $W$ is a Hilbert space. The physical observables are Hermitian (linear) operators in $W$.

(ii) The Hamiltonian equations (1) and the Heisenberg equations (2) are identical (as operator equations) in $W$.

(iii) The projections of the angular momentum of the oscillator $\mathbf{M}=\left(M_{1}, M_{2}, M_{3}\right)$ are in the enveloping algebra of the position operators $\mathbf{r}=\left(r_{1}, r_{2}, r_{3}\right)$ and the momentum operators $\mathbf{p}=\left(p_{1}, p_{2}, p_{3}\right)$. Each $M_{k}$ is linear in $\left(r_{1}, r_{2}, r_{3}\right)$ and linear in $\left(p_{1}, p_{2}, p_{3}\right)$, so that $\mathbf{M}, \mathbf{r}$ and $\mathbf{p}$ transform as vectors:

$$
\left[M_{j}, c_{k}\right]=i \sum_{l=1}^{3} \varepsilon_{j k l} c_{l}, \quad c_{k}=M_{k}, r_{k}, p_{k}, j, k=1,2,3
$$

We underline that in our approach the operators $\mathbf{r}=\left(r_{1}, r_{2}, r_{3}\right)$ and $\mathbf{p}=\left(p_{1}, p_{2}, p_{3}\right)$ are postulated to be the position and the momentum operators of the oscillator, independently of the fact that they do not satisfy the CCRs.

The mathematical problem that arises is to find the unknown operators $\mathbf{r}=\left(r_{1}, r_{2}, r_{3}\right)$ and $\mathbf{p}=\left(p_{1}, p_{2}, p_{3}\right)$ so that the quantum conditions (i)-(iii) hold. To this end it is convenient to pass to new unknown operators 


$$
a_{k}^{ \pm}=\sqrt{\frac{m \omega}{2 \hbar}} r_{k} \mp \frac{i}{\sqrt{2 m \omega \hbar}} p_{k}, \quad k=1,2,3 .
$$

For the sake of convenience we refer to the operators $a_{1}^{ \pm}, a_{2}^{ \pm}, a_{3}^{ \pm}$as to creation and annihilation operators (CAOs). These operators should not be confused with the Bose operators. They are unknown operators we are searching for. Only in one particular representation, corresponding to the canonical case, (5) are Bose CAOs. In terms of $a_{k}^{ \pm}$the Hamiltonian (3) reads:

$$
H=\frac{1}{2} \omega \hbar \sum_{k=1}^{3}\left\{a_{k}^{+}, a_{k}^{-}\right\} .
$$

The condition (ii) yields $(k=1,2,3)$ :

$$
\sum_{i=1}^{3}\left[\left\{a_{i}^{+}, a_{i}^{-}\right\}, a_{k}^{ \pm}\right]= \pm 2 a_{k}^{ \pm}
$$

and it is equivalent to the requirement that the Hamiltonian (in units $\omega \hbar$ ), namely $N=(\omega \hbar)^{-1} H$ is a number operator,

$$
\left[N, a_{k}^{ \pm}\right]= \pm a_{k}^{ \pm}
$$

The equations (7) are a unique consequence from the Hamiltonian equations (1) and the Heisenberg equations (2) independently of the properties of the unknown CAOs $a_{k}^{ \pm}$. They are equal time relations, the time dependence being

$$
a_{k}^{ \pm}(t)=e^{ \pm i \omega t} a_{k}^{ \pm}, \quad a_{k}^{ \pm}(0) \equiv a_{k}^{ \pm}, \quad k=1,2,3 .
$$

Hence eqs. (7) hold, if they are fulfilled at, say, $t=0$.

Let $F$ be the free unital (=with unity) associative algebra with generators $a_{1}^{ \pm}, a_{2}^{ \pm}, a_{3}^{ \pm}$and relations (7). Any representation of $F$ is a candidate for a Wigner quantum oscillator. Out of all such representations one has to select those for which also conditions (i)-(iii) hold. The set of the solutions is not empty, it contains at least the canonical oscillator solution. The general solution of the problem is however unknown to us. In [1] we have listed three classes of solutions of CAOs fulfilling the compatibility eqs. (7). The third class of operators $a_{1}^{ \pm}, a_{2}^{ \pm}, a_{3}^{ \pm}$are the creation and annihilation operators of the $\operatorname{ssp}(3 / 2)$ oscillator. They are defined with the following relations $(\varepsilon, \xi= \pm$ or $\pm 1, i, j, k=1,2,3)$ : 


$$
\begin{aligned}
& {\left[\left\{a_{i}^{+}, a_{j}^{-}\right\}, a_{k}^{\varepsilon}\right]=\frac{2}{3} \delta_{i k} a_{j}^{\varepsilon}-\frac{2}{3} \delta_{j k} a_{i}^{\varepsilon}+\frac{2}{3} \delta_{i j} \varepsilon a_{k}^{\varepsilon},} \\
& {\left[\left\{a_{i}^{\varepsilon}, a_{i}^{\varepsilon}\right\}, a_{k}^{\varepsilon}\right]=0} \\
& \left\{a_{i}^{\varepsilon}, a_{j}^{\varepsilon}\right\}=0, i \neq j, \\
& \left\{a_{i}^{+}, a_{j}^{-}\right\}=-\left\{a_{j}^{+}, a_{i}^{-}\right\}, i \neq j, \\
& \left\{a_{1}^{\varepsilon}, a_{1}^{\xi}\right\}=\left\{a_{2}^{\varepsilon}, a_{2}^{\xi}\right\}=\left\{a_{3}^{\varepsilon}, a_{3}^{\xi}\right\}
\end{aligned}
$$

In the next section we investigate the algebraic structure of the operators (9) and establish their relation to the $\operatorname{ssp}(3 / 2)$ algebra. To this end we first recall the definition of the Lie superalgebra $\operatorname{osp}(3 / 2)$.

\section{Lie superalgebraic properties of the creation and the annihilation operators (9)}

In a matrix form the orthosymplectic LS $\operatorname{ssp}(3 / 2)$ can be defined as the set of all 5-by-5 matrices of the form [5]

$$
\left(\begin{array}{ccc|cc}
a & 0 & b & x & u \\
0 & -a & c & y & v \\
-c & -b & 0 & z & w \\
-- & -- & -- & -- & -- \\
v & u & w & d & e \\
-y & -x & -z & f & -d
\end{array}\right)
$$

where the nonzero entries are arbitrary complex numbers. The even subalgebra $s o(3) \oplus s p(2)$ consists of all matrices (10), for which $x=y=z=u=v=w=0$, whereas the odd subspace is obtained taking $a=b=c=d=e=f=0$. The product (= the supercommutator) $\llbracket, \rrbracket$ of any two homogeneous elements is (a) a matrix anticommutator between odd matrices and (b) a matrix commutator in all other cases.

The algebra $\operatorname{spp}(3 / 2)$ is generated from its subspace $G$, consisting of all matrices (10), for which $a=d=e=f=x=y=u=v=0[6]$. Let $e_{i j}$ be a 5-by-5 matrix with 1 on the cross of the $i^{t h}$ row and the $j^{\text {th }}$ column and zero elsewhere. The matrices

$$
\begin{aligned}
& c_{0}^{-}=\sqrt{2}\left(e_{23}-e_{31}\right), \quad c_{0}^{+}=\sqrt{2}\left(e_{32}-e_{13}\right), \\
& c_{1}^{-}=\sqrt{2}\left(e_{34}-e_{53}\right), \quad c_{1}^{+}=\sqrt{2}\left(e_{35}+e_{43}\right),
\end{aligned}
$$

constitute a basis in $G$ with even generators (11) and odd generators (12). The other 8 osp $(3 / 2)$ generators are the supercommutators of (11)-(12), 


$$
\llbracket c_{p}^{\xi}, c_{q}^{\eta} \rrbracket=c_{p}^{\xi} c_{q}^{\eta}-(-1)^{p q} c_{q}^{\eta} c_{p}^{\xi}, \quad \xi, \eta= \pm, \quad p, q \in \mathbb{Z}_{2} \equiv(0,1) .
$$

The subspace $G$ is a Lie-super triple system in the terminology of Okubo [7]. It is closed under double supercommutators,

$$
\llbracket \llbracket x, y \rrbracket, z \rrbracket=2<y\left|z>x-2(-1)^{\operatorname{deg}(x) \operatorname{deg}(y)}<x\right| z>y \in G, \forall x, y, z \in G,
$$

where the bilinear form $\langle x \mid y\rangle$ is defined as [7]

$$
<c_{p}^{\xi} \mid c_{q}^{\eta}>=\eta^{p} \delta_{p q} \delta_{\xi,-\eta}, \quad \xi, \eta= \pm, \quad p, q \in \mathbb{Z}_{2} \equiv(0,1) .
$$

In terms of the basis (11)-(12) in $G$ eq. (14) reads:

$$
\llbracket \llbracket c_{p}^{\xi}, c_{q}^{\eta} \rrbracket, c_{r}^{\varepsilon} \rrbracket=2 \varepsilon^{r} \delta_{q r} \delta_{\varepsilon,-\eta} c_{p}^{\xi}-2 \varepsilon^{r}(-1)^{q r} \delta_{p r} \delta_{\varepsilon,-\xi} c_{q}^{\eta}, \quad \xi, \eta, \varepsilon= \pm, \quad p, q, r \in \mathbb{Z}_{2}
$$

In another form the eq. (16) was derived in [6]. There it was shown that $B^{ \pm}=c_{1}^{ \pm}$are paraBose operators [8], whereas the operators $F^{ \pm}=c_{0}^{ \pm}$are para-Fermi operators [8]. Observe that the para-Fermi operators appear as even (i.e. bosonic) variables, whereas the parabosons are odd (i.e. fermionic) operators. Moreover the parabosons do not commute with the parafermions. To the same conclusion arrived recently also Okubo [7] and Macfarlane [9]. It may be interesting to observe that the eqs. (16) are satisfied with ordinary bosons and fermions, provided that the bosons anticommute with the fermions [6]. In this way one obtains the simplest infinite-dimensional representation of $\operatorname{osp}(3 / 2)$.

The eqs. (16) were derived using the 5-dimensional representation (10) of $\operatorname{sep}(3 / 2)$. Since however during the derivation we were using only supercommutation relations, eqs. (16) hold within every representation. Therefore from now on (without changing the notation) we consider $c_{p}^{\xi}$ ( $p=$ $0,1 ; \xi= \pm)$ as abstract, representation independent generators. It is essential to point out that the supercommutation relations between all generators $c_{p}^{\xi}, \llbracket c_{p}^{\xi}, c_{q}^{\eta} \rrbracket=c_{p}^{\xi} c_{q}^{\eta}-(-1)^{p q} c_{q}^{\eta} c_{p}^{\xi}(\xi, \eta= \pm ; p, q=$ $0,1)$ can be computed using only eqs. (16). Therefore from the very definition of an universal enveloping algebra we draw our first conclusion.

Proposition 1. The free associative unital algebra $F_{c}$ of the paraoperators $c_{p}^{\xi}(p=0,1 ; \xi= \pm)$ and the relations (16) is the universal enveloping algebra $U[o s p(3 / 2]$ of the Lie superalgebra $o s p(3 / 2)$. The $\mathbb{Z}_{2}$ grading on $U\left[o s p(3 / 2]\right.$ is induced from the requirement that $c_{0}^{ \pm}$are even generators, whereas $c_{1}^{ \pm}$are odd generators.

Define the following 6 elements from $F_{c}$ :

$$
a_{1}^{\varepsilon}=\frac{1}{2 \sqrt{3}}\left[c_{1}^{\varepsilon}, c_{0}^{-}-c_{0}^{+}\right], \quad a_{2}^{\varepsilon}=\frac{i}{2 \sqrt{3}}\left[c_{1}^{\varepsilon}, c_{0}^{-}+c_{0}^{+}\right], \quad a_{3}^{\varepsilon}=\frac{1}{\sqrt{3}} c_{1}^{\varepsilon}, \quad \varepsilon= \pm .
$$


It is straightforward to check that the operators (17) satisfy the relations (9). Let $F_{3}(3)$ be the associative subalgebra of $F_{c}$, generated by the odd elements $a_{1}^{ \pm}, a_{2}^{ \pm}, a_{3}^{ \pm}, F_{3}(3) \subset F_{c}=U[\operatorname{osp}(3 / 2]$. Using only the relations (9), one derives

$$
c_{0}^{\varepsilon}=\frac{3}{2}\left[\varepsilon\left\{a_{1}^{-}, a_{3}^{+}\right\}+i\left\{a_{2}^{-}, a_{3}^{+}\right\}\right]=\frac{3}{2}\left[-\varepsilon\left\{a_{1}^{+}, a_{3}^{-}\right\}-i\left\{a_{2}^{+}, a_{3}^{-}\right\}\right], \quad c_{1}^{\varepsilon}=\sqrt{3} a_{3}^{\varepsilon}, \quad \varepsilon= \pm .
$$

Hence the operators $a_{1}^{ \pm}, a_{2}^{ \pm}, a_{3}^{ \pm}$generate the algebra $F_{c}$,

$$
F_{3}(3)=F_{c}=U[\operatorname{osp}(3 / 2]
$$

Thus, we have proved the result, announced in [1], namely

Proposition 2. The free associative unital algebra $F_{3}(3)$ of the CAOs $a_{1}^{ \pm}, a_{2}^{ \pm}, a_{3}^{ \pm}$and the relations (9) is the universal enveloping algebra $U[\operatorname{sos}(3 / 2]$ of the Lie superalgebra $\operatorname{osp}(3 / 2)$. The $\mathbb{Z}_{2}$ grading on $U[\operatorname{osp}(3 / 2]$ is induced from the requirement that the creation and the annihilation operators are odd elements.

$U[\operatorname{osp}(3 / 2]$ can certainly be viewed as a Lie superalgebra with a supercommutator defined as in every associative superalgebra, namely $\llbracket x, y \rrbracket=x y-(-1)^{\operatorname{deg}(x) \operatorname{deg}(y)} y x$. The linear envelope of $c_{p}^{\xi}, \llbracket c_{p}^{\xi}, c_{q}^{\eta} \rrbracket, \quad \xi, \eta= \pm, \quad p, q=0,1$ is then a Lie subalgebra of the $\operatorname{LS} U[\operatorname{osp}(3 / 2]$ isomorphic to $o s p(3 / 2)$. From (17) and (18) one concludes that (in the category of the Lie superalgebras) the CAOs $a_{1}^{ \pm}, a_{2}^{ \pm}, a_{3}^{ \pm}$generate the subalgebra $\operatorname{osp}(3 / 2)$ of the LS $U[\operatorname{osp}(3 / 2]$. More precisely,

$$
\text { lin.env. }\left\{\left\{a_{i}^{\xi}, a_{j}^{\eta}\right\}, a_{k}^{\varepsilon} \mid \xi, \eta, \varepsilon= \pm, i, j, k=1,2,3\right\}=\operatorname{osp}(3 / 2) .
$$

\section{Satisfying the quantum conditions}

In view of the results of Sec. 2 we already know that any state space $W$ of the CAOs (9) is an osp $(3 / 2)$ module (=representation space of the LS osp $(3 / 2)$ ). The problem is to select those modules, for which the quantum conditions (i)-(iii) hold.

\section{Condition (ii)}

Let $W$ be any $o s p(3 / 2))$ module, i.e., a representation space where the $o s p(3 / 2)$ creation and annihilation operators (9) are defined as linear operators. From eq. (8) one obtains:

$$
r_{k}(t)=\sqrt{\frac{\hbar}{2 m \omega}}\left[a_{k}^{+} e^{i \omega t}+a_{k}^{-} e^{-i \omega t}\right], \quad p_{k}(t)=i \sqrt{\frac{m \omega \hbar}{2}}\left[a_{k}^{+} e^{i \omega t}-a_{k}^{-} e^{-i \omega t}\right] .
$$


It is straightforward to check that the Hamiltonian equations (1) and the Heisenberg equations (2) hold and are identical as operator equations. Hence condition (ii) puts no restriction on the $\operatorname{osp}(3 / 2)$ modules, it holds within each such module. Already now we can say, that if the operators (21) fulfilling all quantum conditions exist, then they possess quite unusual properties. In particular from (9) and (21) one derives that the different coordinates (resp. the different momenta) of the oscillator anticommute:

$$
\left\{r_{i}, r_{j}\right\}=\left\{p_{i}, p_{j}\right\}=0 \quad \forall i \neq j=1,2,3
$$

\section{Condition (iii)}

Consider the operators (21), defined as linear operators in an arbitrary osp(3/2)) representation space $W$. Then the projections $M_{1}, M_{2}$ and $M_{3}$ of the angular momentum can be defined to be:

$$
M_{i}=-\frac{3}{4 \hbar} \sum_{j, k=1}^{3} \varepsilon_{i j k}\left\{r_{j}, p_{k}\right\} \quad i=1,2,3 .
$$

In order to check that eqs. (4) hold it is better to express the angular momentum components in terms of the CAOs (9), namely

$$
M_{j}=-\frac{3 i}{4} \sum_{k, l=1}^{3} \varepsilon_{j k l}\left\{a_{k}^{-}, a_{l}^{+}\right\} \quad j=1,2,3
$$

or in terms of the Lie-super triple generators $c_{p}^{\xi}(p=0,1 ; \xi= \pm)$

$$
M_{1}=-\frac{1}{2}\left(c_{0}^{+}+c_{0}^{-}\right), \quad M_{2}=\frac{i}{2}\left(c_{0}^{+}-c_{0}^{-}\right), \quad M_{3}=\frac{1}{2}\left[c_{0}^{+}, c_{0}^{-}\right]
$$

The angular momentum projections $M_{1}, M_{2}, M_{3}$ are the generators of the so(3) part of the even subalgebra of $\operatorname{ssp}(3 / 2)$, sitting in the left upper corner of the matrix realization (10). Eqs. (25) give the usual realization of $s o(3)=s l(2)$ in terms of para-Fermi (and hence also in terms of Fermi) operators.

\section{Condition (i)}

So far we have satisfied the quantum conditions (ii) and (iii). These conditions put no restriction on the representation space, they hold within each $\operatorname{osp}(3 / 2)$ module. Passing to the condition (i), we have the first restriction.

Proposition 3. If the $\operatorname{osp}(3 / 2)$ module W, satisfying (i), exists then it is completely reducible. 
The proof is standart. Indeed, assume that $E \subset W$ is a subspace of the Hilbert space $W$, which is invariant with respect to the Hermitian operators $a \in\left(r_{1}, r_{2}, r_{3}, p_{1}, p_{2}, p_{3}\right)$. Denote by $F$ its orthogonal compliment, $W=E \oplus F$. Let $e_{1}, \ldots, e_{n}, \ldots$ be an orthonormed basis in $E$ and $f_{1}, \ldots, f_{n}, \ldots$ be an orthonormed basis in $F$. If

$$
a e_{m}=\sum_{p} \alpha_{p m} e_{p}, \quad \text { and } \quad a f_{n}=\sum_{q} \beta_{q n} f_{q}+\sum_{r} \gamma_{r n} e_{r}
$$

then, since $\left(a e_{m}, f_{n}\right)=\left(e_{m}, a f_{n}\right)$, one immediately derives that $\gamma_{r n}=0$ for all values of $r$ and $n$. Hence $F$ is also an invariant subspace. Thus, the orthogonal compliment to each invariant subspace is also an invariant subspace and therefore $W$ is completely reducible. In view of this result the problem reduces to the determination of all irreducible $\operatorname{sep}(3 / 2)$ modules satisfying (i).

The position and the momentum operators are Hermitian operators (hence also the Hamiltonian $H$, the square of the angular momentum $\mathbf{M}^{2}$ and its projections $M_{1}, M_{2}, M_{3}$ are Hermitian operators) if and only

$$
\left(a_{k}^{-}\right)^{\dagger}=a_{k}^{+}, \quad k=1,2,3 \quad \text { or equivalently if } \quad\left(c_{p}^{-}\right)^{\dagger}=c_{p}^{+}, \quad p=0,1
$$

where $(a)^{\dagger}$ is the Hermitian conjugate to the operator $a$.

As in the canonical case, one shows that the energy of any such oscillator should be nonnegative. Indeed, if $\psi$ is a normed eigenvector of the Hamiltonian, $H \psi=E \psi,(\psi, \psi)=1$, then, since $p_{i}$ and $r_{i}$ are Hermitian, from (3) one has

$$
E=(\psi, H \psi)=\sum_{i=1}^{3}\left[\frac{1}{2 m}\left(p_{i} \psi, p_{i} \psi\right)+\frac{m \omega^{2}}{2}\left(r_{i} \psi, r_{i} \psi\right)\right]>0
$$

If $\psi_{0}$ is a state corresponding to the ground energy $E_{0}$ then

$$
a_{k}^{-} \psi_{0}=0, \quad k=1,2,3,
$$

since otherwise $a_{k}^{-} \psi_{0}$ would correspond to a state with an energy $E_{0}-\omega \hbar$.

The irreducible representations (irreps), for which eqs. (26) and (27) hold (we refer to them as to oscillator representations) have been classified (among several others) by Van der Jeugt [10]. They are star irreps [11] with a highest weight. Recently all such irreps have been explicitly constructed [12]. They are infinite-dimensional. Thus, the condition (i) and hence also all conditions (i)-(iii) are satisfied with the oscillator representations of the LS $\operatorname{ssp}(3 / 2)$, which we now proceed to describe.

The oscillator representations are labelled with the set of all possible pairs $(p, q)$, where $p$ is an arbitrary nonnegative half-integer, $p=0,1 / 2,1,3 / 2, \ldots$ and $q$ is any negative real number, such that $p+2 q \leq 0$. The $\operatorname{osp}(3 / 2)$ module corresponding to such a pair is denoted as $W(p, q)$. Each such module is a direct sum of no more than 8 irreducible infinite-dimensional modules $V(p, q ; M, J)$ of 
the even subalgebra $s o(3) \oplus s p(2) . M$ is the angular momentum of the oscillator in a state $\psi$ from $V(p, q ; M, J)$

$$
\mathbf{M}^{2} \psi \equiv\left[\left(M_{1}\right)^{2}+\left(M_{2}\right)^{2}+\left(M_{3}\right)^{2}\right] \psi=M(M+1) \psi \quad \forall \psi \in V(p, q ; M, J)
$$

and $J$ is the analog of $M$ for the subalgebra $s p(2)$, sitting in the right lower corner of matrix representations (10). More precisely, let

$$
J^{ \pm} \equiv J_{1} \pm i J_{2}=\mp \frac{1}{2}\left(c_{1}^{\mp}\right)^{2}, \quad J_{3}=-\frac{1}{4}\left\{c_{1}^{+}, c_{1}^{-}\right\} .
$$

Then $J^{+}$and $J^{-}$are the positive and negative root vectors of $\operatorname{sp}(2)=\operatorname{sl}(2) ; J_{3}$ is the Cartan generator,

$$
\left[J_{3}, J^{ \pm}\right]= \pm J^{ \pm}, \quad\left[J^{+}, J^{-}\right]=2 J_{3}
$$

Eqs. (29) give the usual realization of $s l(2)$ in terms of para-Bose (and hence also in terms of Bose) operators. The label $J$ is the "spin" of the reducible $\operatorname{sp}(2)$ module $V(p, q ; M, J)$,

$$
\mathbf{J}^{2} \psi \equiv\left[\left(J_{1}\right)^{2}+\left(J_{2}\right)^{2}+\left(J_{3}\right)^{2}\right] \psi=J(J+1) \psi \quad \forall \psi \in V(p, q ; M, J)
$$

Let

$$
\theta(x)= \begin{cases}0, & \text { for } x<0 \\ 1, & \text { for } x \geq 0\end{cases}
$$

Then the decomposition of the irreducible $\operatorname{ssp}(3 / 2)$ module $W(p, q)$ into a direct sum of irreducible $s o(3) \oplus \operatorname{sp}(2)$ modules $V(p, q ; M, J)$ reads [12]

$$
\begin{aligned}
& W(p, q)=V(p, q ; p, q) \oplus \theta(p-1) V\left(p, q ; p-1, q-\frac{1}{2}\right) \oplus \theta(p-1) V(p, q ; p-1, q-1) \\
& \oplus \theta\left(p-\frac{1}{2}\right) V\left(p, q ; p, q-\frac{1}{2}\right) \oplus(p+2 q)\left[\theta\left(p-\frac{1}{2}\right) V(p, q ; p, q-1) \oplus V\left(p, q ; p, q-\frac{3}{2}\right)\right. \\
& \left.\oplus V\left(p, q ; p+1, q-\frac{1}{2}\right) \oplus V(p, q ; p+1, q-1)\right] .
\end{aligned}
$$

The multipliers $p+2 q, \theta\left(p-\frac{1}{2}\right)$ and $\theta(p-1)$ are to indicate that at certain values of $p$ and $q$ some of the terms in the r.h.s. of (32) are not present. For instance at $p+2 q=0$ the last 4 terms in (32) disapear. There can be even less terms if in addition $p=0$ or $p=\frac{1}{2}$. Observe that the $s p(2)$ "spin" $J$ of each $V(p, q ; M, J)$ in $(32)$ takes only negative values. It corresponds to unitarizable infinite-dimensional representations of the noncompact real form $s u(1,1)$ of $s p(2)$.

The basis $\left(\mathbb{Z}_{+}=\right.$all nonnegative integers $)$

$$
\mid p, q ; M, J ; m, j>, \quad m=-M,-M+1, \ldots, M-1, M, \quad j=J-n, \quad n \in \mathbb{Z}_{+}
$$


in $V(p, q ; M, J)$ consists of eigenvectors of the Cartan subalgebra, which is a linear span of $M_{3}$ and $J_{3}$. The transformation of the basis under the action of all $\operatorname{osp}(3 / 2)$ generators is completely defined from its transformation under the action of the Lie-super triple system generators $c_{0}^{ \pm}$and $c_{1}^{ \pm}$. The expressions for the even generators, namely the para-Fermi generators $c_{0}^{ \pm}$, are simple:

$$
\left.c_{0}^{ \pm}|p, q ; M, J ; m, j>=-|(M \mp m)(M \pm m+1)\right|^{1 / 2} \mid p, q ; M, J ; m \pm 1, j>.
$$

The transformations under the action of the odd generators $c_{1}^{ \pm}$, i.e., the para-Bose operators, are more involved. They follow and are in fact simpler then the expressions derived in [12]:

For $p \geq 0$ and $p+2 q \leq 0$

$$
\begin{aligned}
& \left.c_{1}^{\mp}\left|p, q ; p, q ; m, j>=\theta\left(p-\frac{1}{2}\right) m\right| \frac{(2 q-1)( \pm q-j)}{q p(p+1)}\right|^{1 / 2} \mid p, q ; p, q-\frac{1}{2} ; m, j \pm \frac{1}{2}> \\
& +\left|\frac{(p+2 q)( \pm q-j)(p+m+1)(p-m+1)}{q(p+1)(2 p+1)}\right|^{1 / 2} \mid p, q ; p+1, q-\frac{1}{2} ; m, j \pm \frac{1}{2}> \\
& +\theta(p-1)\left|\frac{(p-2 q+1)( \pm q-j)(p-m)(p+m)}{q p(2 p+1)}\right|^{1 / 2} \mid p, q ; p-1, q-\frac{1}{2} ; m, j \pm \frac{1}{2}>
\end{aligned}
$$

For $p \geq 0$ and $p+2 q<0$

$$
\begin{aligned}
& c_{1}^{\mp}\left|p, q ; p+1, q-\frac{1}{2} ; m, j>=-\frac{m}{p+1}\right| \pm q-\left.j \mp \frac{1}{2}\right|^{1 / 2} \mid p, q ; p+1, q-1 ; m, j \pm \frac{1}{2}> \\
& -\frac{1}{p+1}\left|\frac{p(p-2 q+1)\left( \pm q-j \mp \frac{1}{2}\right)(p+m+1)(p-m+1)}{q(2 p+1)}\right|^{1 / 2} \mid p, q ; p, q-1 ; m, j \pm \frac{1}{2}> \\
& +\left|\frac{(p+2 q)\left(\mp q-j \mp \frac{1}{2}\right)(p+m+1)(p-m+1)}{q(p+1)(2 p+1)}\right|^{1 / 2} \mid p, q ; p, q ; m, j \pm \frac{1}{2}>
\end{aligned}
$$

For $p>0$ and $p+2 q \leq 0$

$$
\begin{aligned}
& \left.c_{1}^{\mp}\left|p, q ; p, q-\frac{1}{2} ; m, j>=m\right| \frac{(2 q-1)\left(\mp q-j \mp \frac{1}{2}\right)}{q p(p+1)}\right|^{1 / 2} \mid p, q ; p, q ; m, j \pm \frac{1}{2}> \\
& +\frac{1}{p+1}\left|\frac{2 p(p+2 q)\left( \pm q-j \mp \frac{1}{2}\right)(p+m+1)(p-m+1)}{(2 q-1)(2 p+1)}\right|^{1 / 2} \mid p, q ; p+1, q-1 ; m, j \pm \frac{1}{2}> \\
& -\frac{1}{p}\left|\frac{2(p+1)(p-2 q+1)\left( \pm q-j \mp \frac{1}{2}\right)(p+m)(p-m)}{(2 q-1)(2 p+1)}\right|^{1 / 2} \mid p, q ; p-1, q-1 ; m, j \pm \frac{1}{2}> \\
& +\frac{m}{p(p+1)}\left|\frac{(p-2 q+1)(p+2 q)\left( \pm q-j \mp \frac{1}{2}\right)}{q(2 q-1)}\right|^{1 / 2} \mid p, q ; p, q-1 ; m, j \pm \frac{1}{2}>
\end{aligned}
$$

For $p \geq 1$ and $p+2 q \leq 0$

$$
c_{1}^{\mp}\left|p, q ; p-1, q-\frac{1}{2} ; m, j>=\frac{m}{p}\right| 2\left( \pm q-\left.j \mp \frac{1}{2}\right|^{1 / 2} \mid p, q ; p-1, q-1 ; m, j \pm \frac{1}{2}>\right.
$$




$$
\begin{aligned}
& +\left|\frac{(p-2 q+1)\left(\mp q-j \mp \frac{1}{2}\right)(p-m)(p+m)}{q p(2 p+1)}\right|^{1 / 2} \mid p, q ; p, q ; m, j \pm \frac{1}{2}> \\
& +\frac{1}{p}\left|\frac{(p+2 q)(p+1)\left( \pm q-j \mp \frac{1}{2}\right)(p+m)(p-m)}{q(2 p+1)}\right|^{1 / 2} \mid p, q ; p, q-1 ; m, j \pm \frac{1}{2}>,
\end{aligned}
$$

For $p \geq 0$ and $p+2 q<0$

$$
\begin{aligned}
& \left.c_{1}^{\mp}\left|p, q ; p+1, q-1 ; m, j>=-\frac{m}{p+1}\right| 2(\mp q-j)\right|^{\frac{1}{2}} \mid p, q ; p+1, q-\frac{1}{2} ; m, j \pm \frac{1}{2}> \\
& +\frac{1}{p+1}\left|\frac{2 p(p+2 q)(\mp q-j)(p+m+1)(p-m+1)}{(2 q-1)(2 p+1)}\right|^{1 / 2} \mid p, q ; p, q-\frac{1}{2} ; m, j \pm \frac{1}{2}> \\
& +\left|\frac{2(p-2 q+1)( \pm q-j \mp 1)(p+m+1)(p-m+1)}{(2 q-1)(p+1)(2 p+1)}\right|^{1 / 2} \mid p, q ; p, q-\frac{3}{2} ; m, j \pm \frac{1}{2}>
\end{aligned}
$$

For $p>0$ and $p+2 q<0$

$$
\begin{aligned}
& \left.c_{1}^{\mp}|p, q ; p, q-1 ; m, j>=-2 m| \frac{q( \pm q-j \mp 1)}{p(p+1)(2 q-1)}\right|^{1 / 2} \mid p, q ; p, q-\frac{3}{2} ; m, j \pm \frac{1}{2}> \\
& +\frac{m}{p(p+1)}\left|\frac{(p-2 q+1)(p+2 q)(\mp q-j)}{q(2 q-1)}\right|^{1 / 2} \mid p, q ; p, q-\frac{1}{2} ; m, j \pm \frac{1}{2}> \\
& -\frac{1}{p+1}\left|\frac{p(p-2 q+1)(\mp q-j)(p+m+1)(p-m+1)}{q(2 p+1)}\right|^{1 / 2} \mid p, q ; p+1, q-\frac{1}{2} ; m, j \pm \frac{1}{2}> \\
& +\frac{1}{p}\left|\frac{(p+2 q)(p+1)(\mp q-j)(p+m)(p-m)}{q(2 p+1)}\right|^{1 / 2} \mid p, q ; p-1, q-\frac{1}{2} ; m, j \pm \frac{1}{2}>,
\end{aligned}
$$

For $p \geq 1$ and $p+2 q \leq 0$

$$
\begin{aligned}
& \left.c_{1}^{\mp}\left|p, q ; p-1, q-1 ; m, j>=\frac{m}{p}\right| 2(\mp q-j)\right|^{\frac{1}{2}} \mid p, q ; p-1, q-\frac{1}{2} ; m, j \pm \frac{1}{2}> \\
& -\frac{1}{p}\left|\frac{2(p-2 q+1)(p+1)(\mp q-j)(p+m)(p-m)}{(2 q-1)(2 p+1)}\right|^{1 / 2} \mid p, q ; p, q-\frac{1}{2} ; m, j \pm \frac{1}{2}> \\
& +\left|\frac{2(p+2 q)( \pm q-j \mp 1)(p+m)(p-m)}{p(2 q-1)(2 p+1)}\right|^{1 / 2} \mid p, q ; p, q-\frac{3}{2} ; m, j \pm \frac{1}{2}>,
\end{aligned}
$$

For $p \geq 0$ and $p+2 q<0$

$$
\begin{aligned}
& \left.c_{1}^{\mp}\left|p, q ; p, q-\frac{3}{2} ; m, j>=-\theta\left(p-\frac{1}{2}\right) 2 m\right| \frac{q\left(\mp q-j \pm \frac{1}{2}\right)}{p(p+1)(2 q-1)}\right|^{1 / 2} \mid p, q ; p, q-1 ; m, j \pm \frac{1}{2}> \\
& +\left|\frac{2(p-2 q+1)\left(\mp q-j \pm \frac{1}{2}\right)(p+m+1)(p-m+1)}{(2 q-1)(p+1)(2 p+1)}\right|^{1 / 2} \mid p, q ; p+1, q-1 ; m, j \pm \frac{1}{2}> \\
& \theta(p-1)\left|\frac{2(p+2 q)\left(\mp q-j \pm \frac{1}{2}\right)(p+m)(p-m)}{p(2 q-1)(2 p+1)}\right|^{1 / 2} \mid p, q ; p-1, q-1 ; m, j \pm \frac{1}{2}>
\end{aligned}
$$

The above eqs. (34)-(42) are not easy to be derived. It is even quite difficult to verify that they give a representation of the Lie-super triple relations (16). In doing so and, more generally, applying 
eqs. (35)-(42) one should have in mind that whenever a $\theta$-function in front of a certain term in the r.h.s. vanishes, then the corresponding term should be canceled out independently of the fact that some other multipliers in the same term could be undefined (at $p=0$ one has sometimes factors $\frac{0}{0}$ ).

The transformation relations of the basis under the action of some even generators, which follow from (33)-(42), read:

$$
\begin{aligned}
& s o(3) \text { generators } M^{ \pm}=M_{1} \pm i M_{2}, M_{3}: \\
& \left.M^{ \pm}|p, q ; M, J ; m, j>=|(M \mp m)(M \pm m+1)\right|^{1 / 2} \mid p, q ; M, J ; m \pm 1, j> \\
& M_{3}|p, q ; M, J ; m, j>=m| p, q ; M, J ; m, j>, \\
& \mathbf{M}^{2}|p, q ; M, J ; m, j>=M(M+1)| p, q ; M, J ; m, j>; \\
& s p(2) \text { generators : } \\
& \left.J^{ \pm}|p, q ; M, J ; m, j>=|(J \pm j+1)(J \mp j)\right|^{1 / 2} \mid p, q ; M, J ; m, j \pm 1>, \\
& J_{3}|p, q ; M, J ; m, j>=j| p, q ; M, J ; m, j>. \\
& \mathbf{J}^{2}|p, q ; M, J ; m, j>=J(J+1)| p, q ; M, J ; m, j>;
\end{aligned}
$$

Postulate that the basis (33) within each $s o(3) \oplus \operatorname{sp}(2)$ module $V(p, q ; M, J)$ is orthonormed and that the different such modules are orthogonal to each other. With respect to this metric the hermiticity conditions (26) hold. Consequently also $\left(r_{1}, r_{2}, r_{3}\right),\left(p_{1}, p_{2}, p_{3}\right), \mathbf{M}^{2},\left(M_{1}, M_{2}, M_{3}\right)$ and $H$ are Hermitian operators. Thus the condition (i) and hence all quantum conditions (i)-(iii) are satisfied within any oscillator module $W(p, q)$, considered as a state space of a noncanonical oscillator. Hence any triple $(\mathbf{r}, \mathbf{p}, W(p, q))$ is a Wigner quantum oscillator.

\section{Energy and angular momentum spectrum of the oscillators. Multiplicities}

The Hamiltonian (3) of the $\operatorname{osp}(3 / 2)$ oscillator is proportional to $J_{3}$ (see $\left.(29)\right)$,

$$
H=\frac{\omega \hbar}{2}\left\{c_{1}^{+}, c_{1}^{-}\right\}=-2 \omega \hbar J_{3} \in \operatorname{sp}(2) .
$$

The operators $H, \mathbf{M}^{2}$ and $M_{3}$ commute with each other. According to (44), (45), (47) and (49) the basis (33) is diagonal with respect to these operators. In particular

$$
H|p, q ; M, J ; m, j>=-2 \omega \hbar j| p, q ; M, J ; m, j>
$$

Each state space $W(p, q)$ contains a subspace $V(p, q ; M, J)$ with $J=q$ and at least one subspace with $J=q-\frac{1}{2}$. Taking into account that within $V(p, q ; M, J) j=J, J-1, J-2, \ldots$ from (50) one obtains the spectrum $E_{n}$ of $H$ in the subspaces with $J=q$ and $J=q-\frac{1}{2}$ : 


$$
\begin{gathered}
\text { In } V(p, q ; p, q) \quad E_{n}=\omega \hbar(2 n-2 q), \quad n=0,1,2,3, \ldots ; \\
\text { In } V\left(p, q ; M, q-\frac{1}{2}\right) \quad E_{n}=\omega \hbar[(2 n+1)-2 q], \quad n=0,1,2,3, \ldots .
\end{gathered}
$$

Combining (51) and (52) one obtains

$$
E_{n}=\omega \hbar(n-2 q), \quad n=0,1,2,3, \ldots
$$

The energies of the states in the other subspaces $V(p, q ; M, J)$ do not change the spectrum (53); they change only the multiplicities of the energy levels. Therefore eq. (53) gives the energy levels of the oscillator in a state space $W(p, q)$

From the decomposition (32) and eq. (28) one concludes that the angular momentum of an $\operatorname{osp}(3 / 2)$ oscillator in a state space $W(p, q)$ can take at most three different values, namely

$$
M=p-1, p, p+1
$$

The states from a subspace $V(p, q ; M, J)$ carry an angular momentum $M$. Each such space is infinite-dimensional. Hence the multiplicity of each allowed value $M$ of the angular momentum is also infinite-dimensional.

In order to analyze the multiplicities of the stationary states, we first observe that each irreducible $s o(3) \oplus \operatorname{sp}(2)$ module $V(p, q ; M, J)$ is a tensor product of an irreducible $2 M+1$ dimensional so(3) module $[M]$ with an irreducible infinite-dimensional $s p(2)$ module $[J]$,

$$
V(p, q ; M, J)=[M] \otimes[J]
$$

An operator $a$ from $s o(3)$ acts in $[M] \otimes[J]$ as $a \otimes i d$, whereas an operator $b$ from $s p(2)$ acts as $i d \otimes b$ ( $i d=$ identity operator). Each basis vector $\mid p, q ; M, J ; m, j>\in V(p, q ; M, J)$ can be represented as

$$
|p, q ; M, J ; m, j>=| M, m>\otimes \mid J, j>
$$

Then

$$
\begin{aligned}
& \mathbf{M}^{2}|M, m>=M(M+1)| M, m>, \quad M_{3}|M, m>=m| M, m> \\
& \mathbf{J}^{2}|J, j>=J(J+1)| J, j>, \quad J_{3}|J, j>=j| J, j>
\end{aligned}
$$

From (54) it is evident that the linear envelope of all $\mid p, q ; M, J ; m, j>$ states from $V(p, q ; M, J)$ with a fixed $j$, namely with a fixed energy, is an irreducible $(2 M+1)$ dimensional so(3) module $[M]$. This observation together with the decomposition (32) leads to the conclusion that the eigenspace $V\left(E_{n}\right)$ of the Hamiltonian $H$ in $W(p, q)$ is generally reducible so(3) module. 
Let

$$
\varphi(x)= \begin{cases}1, & \text { for } x=0,1,2, \ldots \\ 0, & \text { otherwise. }\end{cases}
$$

Then for the eigenspace $V\left(E_{n}\right)$ of the Hamiltonian $H$ in $W(p, q)$ we obtain:

$$
\begin{aligned}
& V\left(E_{n}\right)=\theta(p-1) \theta(n-1)[p-1] \oplus\{1-\theta(p+2 q)\} \theta(n-1)[p+1] \oplus\left\{\theta\left(p-\frac{1}{2}\right) \varphi\left(\frac{n-1}{2}\right)\right. \\
& \left.+\varphi\left(\frac{n}{2}\right)+\{1-\theta(p+2 q)\} \theta\left(p-\frac{1}{2}\right) \varphi\left(\frac{n}{2}-1\right)+\{1-\theta(p+2 q)\} \varphi\left(\frac{n-3}{2}\right)\right\}[p] .
\end{aligned}
$$

From eq. (59) one concludes that an oscillator with a state space $W(p, q)$ has

a) $\theta(p-1) \theta(n-1)(2 p-1)$ states with an angular momentum $M=p-1$ and energy $E_{n}$,

b) $\left\{\theta\left(p-\frac{1}{2}\right) \varphi\left(\frac{n-1}{2}\right)+\varphi\left(\frac{n}{2}\right)+\{1-\theta(p+2 q)\} \theta\left(p-\frac{1}{2}\right) \varphi\left(\frac{n}{2}-1\right)+\{1-\theta(p+2 q)\} \varphi\left(\frac{n-3}{2}\right)\right\}(2 p+1)$ states with an angular momentum $M=p$ and energy $E_{n}$,

c) $\{1-\theta(p+2 q)\} \theta(n-1)(2 p+3)$ states with an angular momentum $M=p+1$ and energy $E_{n}$,

Summing up the expressions in a), b) and c) one obtains the number of the (linearly independent) states with energy $E_{n}$, i.e., $\operatorname{dim} V\left(E_{n}\right)$.

Let us consider the cases corresponding to different values of $p$.

\section{Oscillators with $\mathrm{p}=0$}

Since $q<0$, then also $p+2 q<0$ and according to (59)

$$
V\left(E_{0}\right)=[0], \quad V\left(E_{1}\right)=[1], \quad V\left(E_{n}\right)=[0] \oplus[1], n>1 .
$$

In particular the ground state $(n=0)$ is nondegenerated and carries an angular momentum zero. Depending on the value of $q$ the energy of the ground state $E_{0}=-2 \omega \hbar q$ can be arbitrarily close to zero, but never zero. The first excited states $(n=1)$ have $M=1$. In all other cases $(n>1)$ the eigenspace of the Hamiltonian is reducible with respect to so(3). There is one state with angular momentum zero and three spaces with $M=1$.

\section{An oscillator with $p=1 / 2$ and $p+2 q=0$}

This case corresponds to the oscillator considered in [1]. The angular momentum of each state is $M=\frac{1}{2}, V\left(E_{n}\right)=\left[\frac{1}{2}\right]$ for any $n$; there are two states corresponding to every energy level. The

state space $W\left(\frac{1}{2},-\frac{1}{4}\right)$ is an infinite direct sum of 2-dimensional representations of so(3). In this case the expressions (35)-(42) are greatly simplified; $c_{0}^{ \pm}$are usual Fermi operators, whereas $c_{1}^{ \pm}$are Bose operators. Moreover the bosons anticommute with the fermions. The representation of $\operatorname{osp}(3 / 2)$ is one of the metaplectic representations of the superalgebra [10]. The energy spectrum of the oscillator is the same as for an one-dimensional canonical harmonic oscillator, 


$$
E_{n}=\omega \hbar\left(n+\frac{1}{2}\right), \quad n=0,1,2, \ldots
$$

\section{Oscillators with $p=1 / 2$ and $p+2 q<0$}

The subspace corresponding to the ground energy is two-dimensional and carries an angular momentum $\frac{1}{2}$. The angular momentum of all other states is either $\frac{1}{2}$ or $\frac{3}{2}$. More precisely one has:

$$
V\left(E_{0}\right)=\left[\frac{1}{2}\right], \quad V\left(E_{1}\right)=\left[\frac{1}{2}\right] \oplus\left[\frac{3}{2}\right], \quad V\left(E_{n}\right)=\left[\frac{1}{2}\right] \oplus\left[\frac{1}{2}\right] \oplus\left[\frac{3}{2}\right], n>1
$$

\section{Oscillators with $\mathrm{p}>1 / 2$ and $\mathrm{p}+2 \mathrm{q}=0$}

The angular momentum of the ground subspace is $M=p$. There are $2 p+1$ ground states; all other states have an angular momentum $p$ or $p-1$ :

$$
V\left(E_{0}\right)=[p], \quad V\left(E_{n}\right)=[p] \oplus[p-1], n \geq 1
$$

\section{Oscillators with $p>1 / 2$ and $p+2 q<0$}

The structure of the ground subspace is the same as in the previous case. The angular momentum of any other eigenspace of the Hamiltonian is a reducible so(3) module with angular momentum $M=p-1, p, p+1$. Its $s o(3)$ content reads:

$V\left(E_{0}\right)=[p], \quad V\left(E_{1}\right)=[p-1] \oplus[p] \oplus[p+1], \quad V\left(E_{n}\right)=[p-1] \oplus[p] \oplus[p] \oplus[p+1], n>1$

\section{Discussions}

From the above considerations it is clear that the $\operatorname{sep}(3 / 2)$ oscillators differ essentially from the canonical 3-dimensional oscillator. We underline some of the main points in this respect.

The coordinates of any $\operatorname{osp}(3 / 2)$ oscillator anticommute with each other (see eq.(22)). Therefore one cannot have a coordinate ( or $x$-) representation for the wave function. The geometry of the oscillator is noncommutative. For the same reason there exists no momentum representation. Here we have considered the case with $H, \mathbf{M}^{2}$ and $M_{3}$ being simultaneously diagonal, namely an energy - angular momentum representation.

The canonical oscillator can be in a state with any integer value of the angular momentum $M$, but never in a state with half-integer values of $M$. An $\operatorname{osp}(3 / 2)$ oscillator allows at most three values 
of the angular momentum, $M=p-1, p, p+1$, but they can be either integers or half-integers. In particular if $p=\frac{1}{2}$ then the angular momentum takes only one value $M=\frac{1}{2}$; if $p=0$ then $M=0,1$; in all other cases $M$ can have three different values as indicated above.

The energy spectrum of any $\operatorname{osp}(3 / 2)$ oscillator is equidistant. In four cases, namely in the state spaces $W\left(p,-\frac{3}{4}\right)$ with $p=0, \frac{1}{2}, 1, \frac{3}{2}$ the spectrum is the same as of the canonical 3-dimensional oscillator,

$$
E_{n}=\omega \hbar\left(n+\frac{3}{2}\right), \quad n=1,2,3, \ldots
$$

In all other cases the spectrum is different. It may be even very different for large values of $p$. Indeed the conditions $p+2 q \leq 0, q<0$ put restrictions from bellow for the ground energy, namely $E_{0} \geq \omega \hbar p$. Even for small values of $p$, but large values of $q$ the ground energy $E_{0}$ may be much above the canonical ground energy $\frac{3}{2} \omega \hbar$.

Another essential new feature we like to point out is the degeneracy of the ground states. As one can see from eq. (60), the eigenspace $V\left(E_{0}\right)$ of the Hamiltonian is nondegenerated only in the state spaces $W(0, q)$, i.e., those with $p=0$. In all cases the states from $V\left(E_{0}\right)$ carry one and the same angular momentum, namely $M=p$ if $V\left(E_{0}\right) \subset W(p, q)$; the ground subspace transforms as an irreducible $s o(3)$ module $V\left(E_{0}\right)=[p]$. In the state spaces with $p \geq 1$ the eigenspaces $V\left(E_{n}\right), n>0$, of $H$ carry different angular momentum, they are reducible with respect to so(3).

As a last remark we mention that all our considerations are in the Heisenberg picture; the operators are generally time dependent. The only time independent operators (from those we have considered) are the Hamiltonian $H$, the square of the angular momentum operator $\mathbf{M}^{2}$ and its projections $\left(M_{1}, M_{2}, M_{3}\right)$, namely the operators generating the stability subalgebra $s o(3) \oplus g l(1)$. The root vectors $J^{ \pm}$of $s p(2)$ and all odd generators (see (8) or (21)) depend on time.

\section{Acknowledgements}

One of us (T. P.) is thankful to Prof. H. D. Doebner for the kind hospitality at the Arnold Sommerfeld Institute for Mathematical Physics, where some of the results in the present investigation have been obtained.

\section{References}

[1] Palev T D and Stoilova N I 1994 J. Phys. A: Math. Gen. 27977

[2] Wigner E P 1950 Phys. Rev. 77711

[3] Ohnuki Y and Kamefuchi S 1978 J. Math. Phys. 19 67; 1979 Z. Phys. C 2367

Okubo S 1980 Phys. Rev. D 22 919; 
Mukunda N, Sudarshan E C G, Sharma J K and Mehta C L 1980 J. Math. Phys. 21 2386;

Ohnuki Y and Watanabe S 1992 J. Math. Phys. 333653

[4] Palev T D 1982 J. Math. Phys. 23 1778; 1982 Czech. J. Phys. B 3680

Kamupingene A H, Palev T D and Tsaneva S P 1986 J. Math. Phys. 272067

[5] Kac V G 1977 Adv. Math. 268

[6] Palev T D 1982 J. Math. Phys. 231100

[7] Okubo S 1993 University of Rochester preprint ER-1335, ERO40685-784

[8] Green H S 1953 Phys. Rev. 90270

[9] Macfarlane A J 1994 J. Math. Phys. 351054

[10] Van der Jeugt J 1984 J. Math. Phys. 253334

[11] Scheunert M, Nahm W and Rittenberg V 1977 J. Math. Phys. 18146

[12] Ky N A, Palev T D and Stoilova N I 1992 J. Math. Phys. 331841 